\title{
Observations on Ascospore Initiation in the Discomycete Dasyscyphus sp.
}

\author{
By R. T. MOORE* AND J. H. MCALEAR* \\ Department of Plant Pathology, Cornell University, Ithaca, New York, and \\ Division of Laboratories and Research, New York State Department of Health, \\ Albany, New York, U.S.A. \\ (Received 6 July 1961) \\ SUMMARY \\ Electron microscopy of apothecia of a species of a discomycete, Dasyscy- \\ phus sp., provided the opportunity to observe various aspects of ascospore \\ formation. This report presents a series of micrographs arranged to repre- \\ sent what are interpreted to be successive stages in the process.
}

\section{INTRODUCTION}

Fungal cytology, unlike that of most plants and animals, lies near the limits of resolution of the light microscope. Consequently the nature of the various cell inclusions and organelles in fungal cells is poorly understood. Within recent years, however, the electron microscope has provided one means for gaining a clearer insight into the micromorphology of the somatic and reproductive structures of these organisms. The purpose of this paper is to show some of the changes in fine structure that occur in the ascus.

\section{METHODS}

Fruiting bodies (apothecia) of a species of a discomycete, Dasyscyphus sp., were collected in the field and prefixed in aqueous unbuffered $2 \%(\mathrm{w} / \mathrm{v}) \mathrm{KMnO}_{4}$ for $9 \mathrm{~min}$. and after briefly rinsing in distilled water were fixed for $5 \mathrm{hr}$. at $4^{\circ}$ in buffered $2 \%(\mathrm{w} / \mathrm{v}) \mathrm{OsO}_{4}$. Subsequently they were rinsed in buffer alone, dehydrated in an ethanol series and embedded in a methacrylate mixture. (Details of this protocol appear in Moore, 1962.) Sections were cut on a Servall Porter-Blum ultramicrotome with a Fernández-Morán diamond knife and examined in a Siemens Elmiskope I electron microscope.

\section{RESULTS}

Within the ascus during ascospore formation the fusion of opposing haploid nuclei is followed immediately by meiosis. Typically in the Ascomycetes, including Dasyscyphus, there is a post-meiotic division to produce a total of eight nuclei. Subsequently these nuclei become encapsulated by wall material to become ascospores. Plate 1 , fig. 1 , is interpreted as a stage in karyogamy and shows a fusion nucleus $(N)$ composed of typical light (electron transparent), coarse granular, and

* Present address: Electron Microscope Laboratory, University of California, Berkeley, California, U.S.A. 
dark (electron dense), fine granulau regions (see McAlear \& Edwards, 1959; Moore, 1962; Moore \& McAlear, 1961). Flate 1, fig. 2, is interpreted as representing an early stage in meiosis. The nuclear :nvelope has broken down and is not discernible; the nucleophase, however, is still frominent but merges indistinguishably into the cytoplasm. Plate 2, fig. 3, shows two masses of material $(N)$, each composed of a coarse granular electron-transparent discrete phase, and an electron-dense fine granular phase that merges with tae ascus cytoplasm. These are interpreted to be two units of nucleoplasm and to represent a stage of meiosis subsequent to that shown in the previous figure. A m mbrane fragment (wedges), observable near the lower moiety, may be a segment of the dissociated nuclear envelope. Plate 2, fig. 4, represents a stage in the reformation of endomembranes. Elements of the endoplasmic reticulum $(E R)$ are prominent and the reconstitution of the nuclear envelopes is well advanced. Plate 3 , fig. 5 , is interpreted as a stage of late telophase. Three of the presumed four nuclei $(N)$ are evident. 'The nuclear envelopes are nearly complete but nuclear separation has not yet occurred as evidenced by the opposing gaps (arrows) in the respective nt clear envelopes. Plate 4, fig. 6, is a much later stage and shows a nearly mature a scus and five of the final eight ascospores. Within the ascospores may be seen typical nuclei $(N)$, mitochondria $(\boldsymbol{M})$ and the endoplasmic reticulum $(\boldsymbol{E R})$. This last (in the upper right spore) appears to form continuities between the nuclear envelope and the plasma membrane. Such continuities are not rare in fungi and have beeı reported in Blastomyces dermatitidis (Edwards \& Edwards, 1960), Stilbum zacalloxc nthum (McAlear \& Edwards, 1959), Mollisia sp. (Moore \& McAlear,1961) and Ascodesmis sphaerospora (Moore, 1962). The cytoplasmic material left outside the spore, exiplasm $(E)$, contains no nuclei and breaks down during spore maturation. The da: $\mathrm{k}$ material bounding the spores is apparently a separable layer (wedges). It may be a residue of the epiplasm or material that has passed out through the spore wall $n$ a manner similar to the formation of the opaque secondary spore coat in Ascodesn is (Moore, 1962).

\section{DISCUSSION}

The sequence of events in ascospore initiation in Dasyscyphus appears to be quite complementary to those rejorted for another discomycete (Moore, 1962). In that report micrographs showed the fusion nucleus, the terminal eight nucleate stage at the end of free nuclear div ision before wall initiation, and ascospore maturation. The present observations show the stages believed to occur between karyogamy and the formation of the final oc ad of nuclei, i.e. during free nuclear division. We have other micrographs which sh w initial wall formation in two other discomycetes (Coryne sp. and Neobulgaria purc); in these the new wall material first appears as a thin electron-transparent shell c ncompassing each of the final nuclei and a portion of the adjoining cytoplasm. Subsequently, the wall becomes much thickened. In Dasyscyphus this is the only spore wall and it appears to be quite similar to the primary wall in Ascodesmis. $B_{y}$ both light and electron microscopy it appears smooth and transparent. By colliting observations from several allied fungi we are able to reconstruct nearly the whole sequence of ascospore formation. However, the over-all rarity of the micrographs that we have been able to obtain of the various steps strongly suggests that the ontogeny occurs quite rapidly. In a number of Ascomycetes spore maturation ir volves further modifications such as the formation 


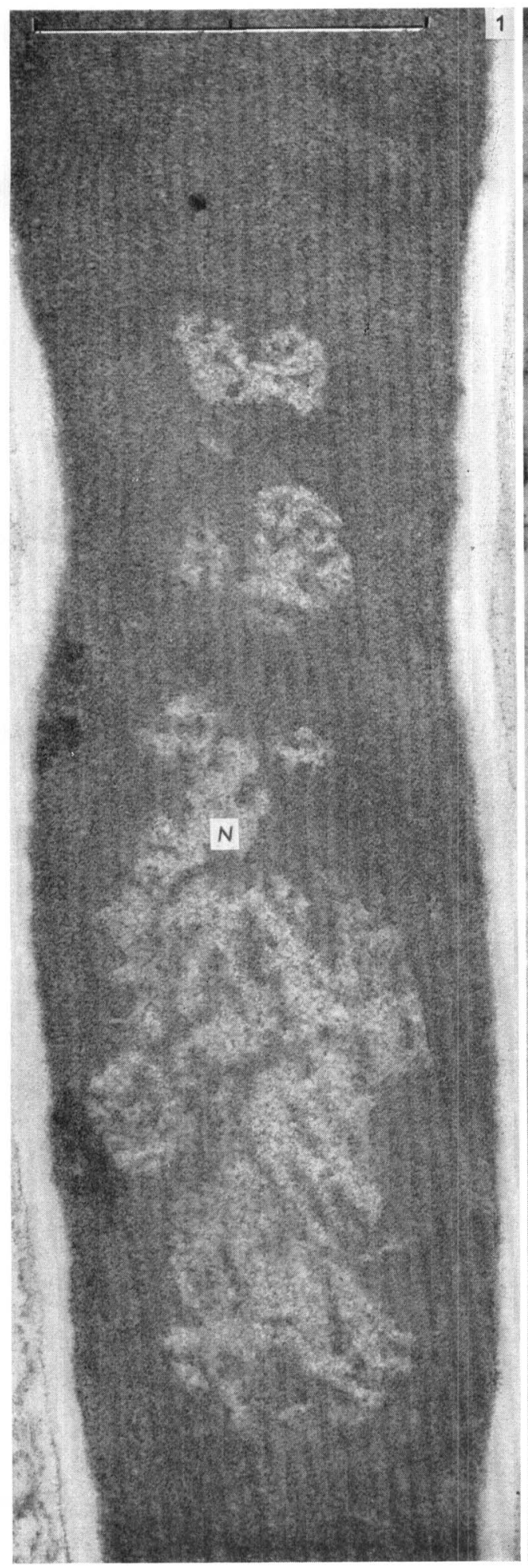

R. T. MOORE AND J. H. MCALEAR

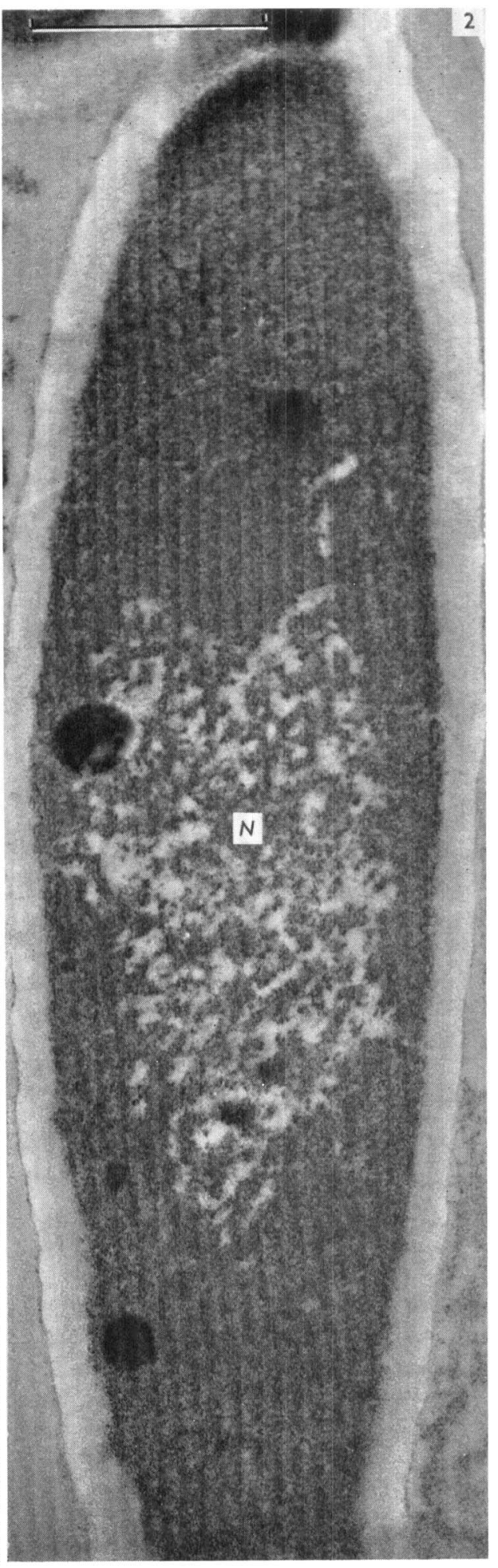

(Facing p. 212) 
Journal of General Microbiology, Vol. 28, No. 2

Plate 2

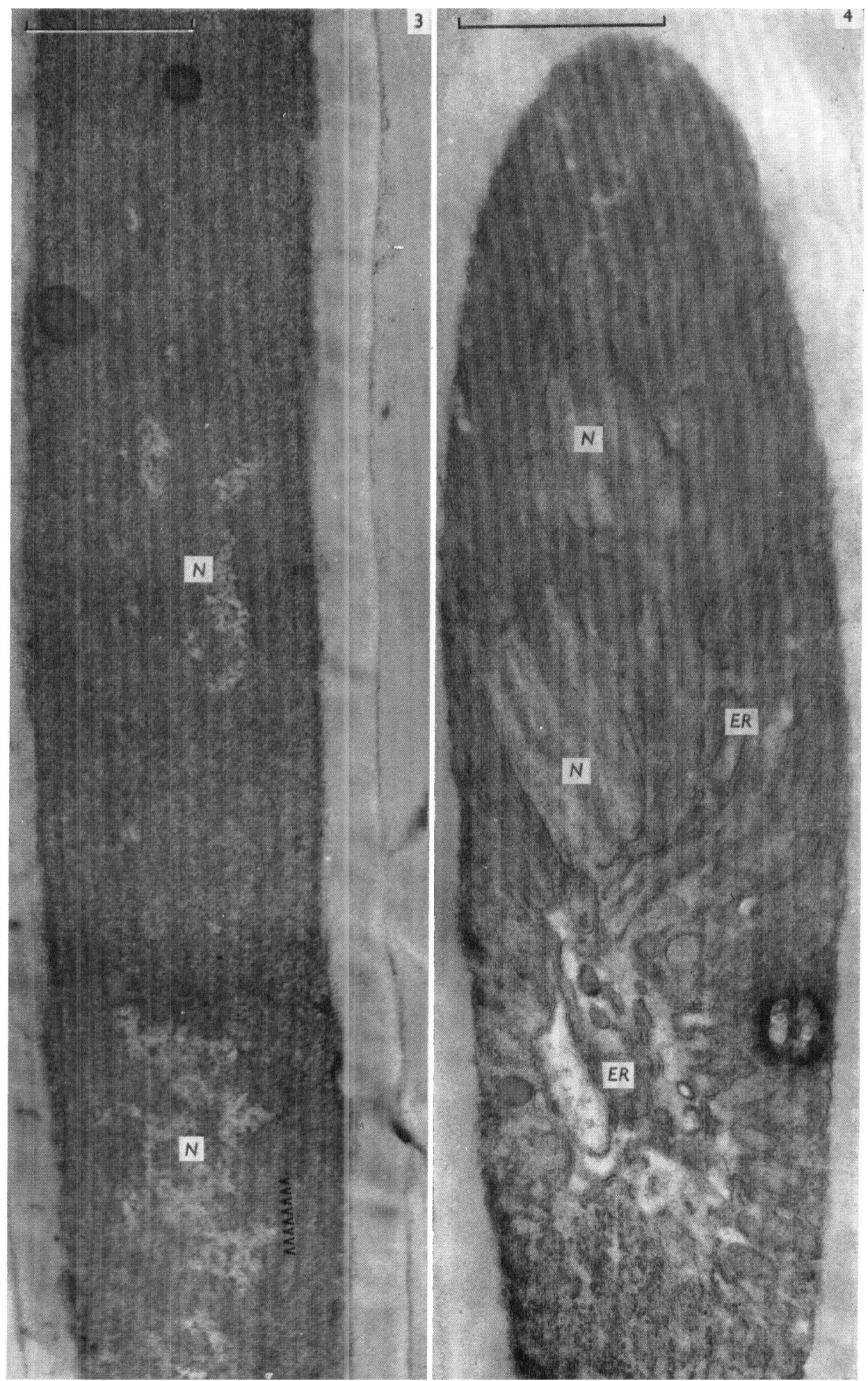

R. T. MOORE AND J. H. MCALEAR 
Journal of General Microbiology, Vol. 28, No. 2

Plate 3

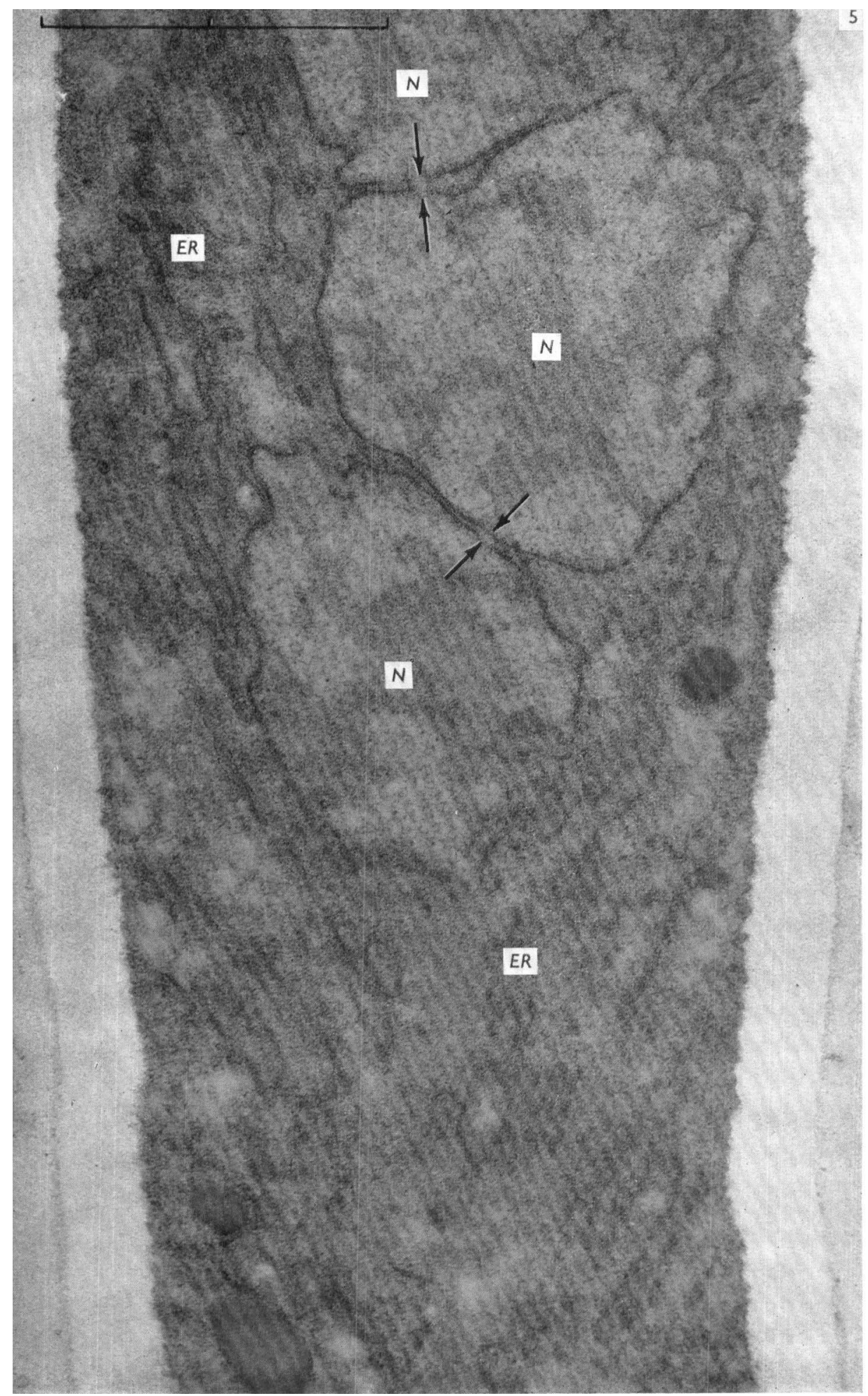

R. T. MOORE AND J. H. MCALEAR 
Journal of General Microbiology, Vol. 28, No. 2

Plate 4

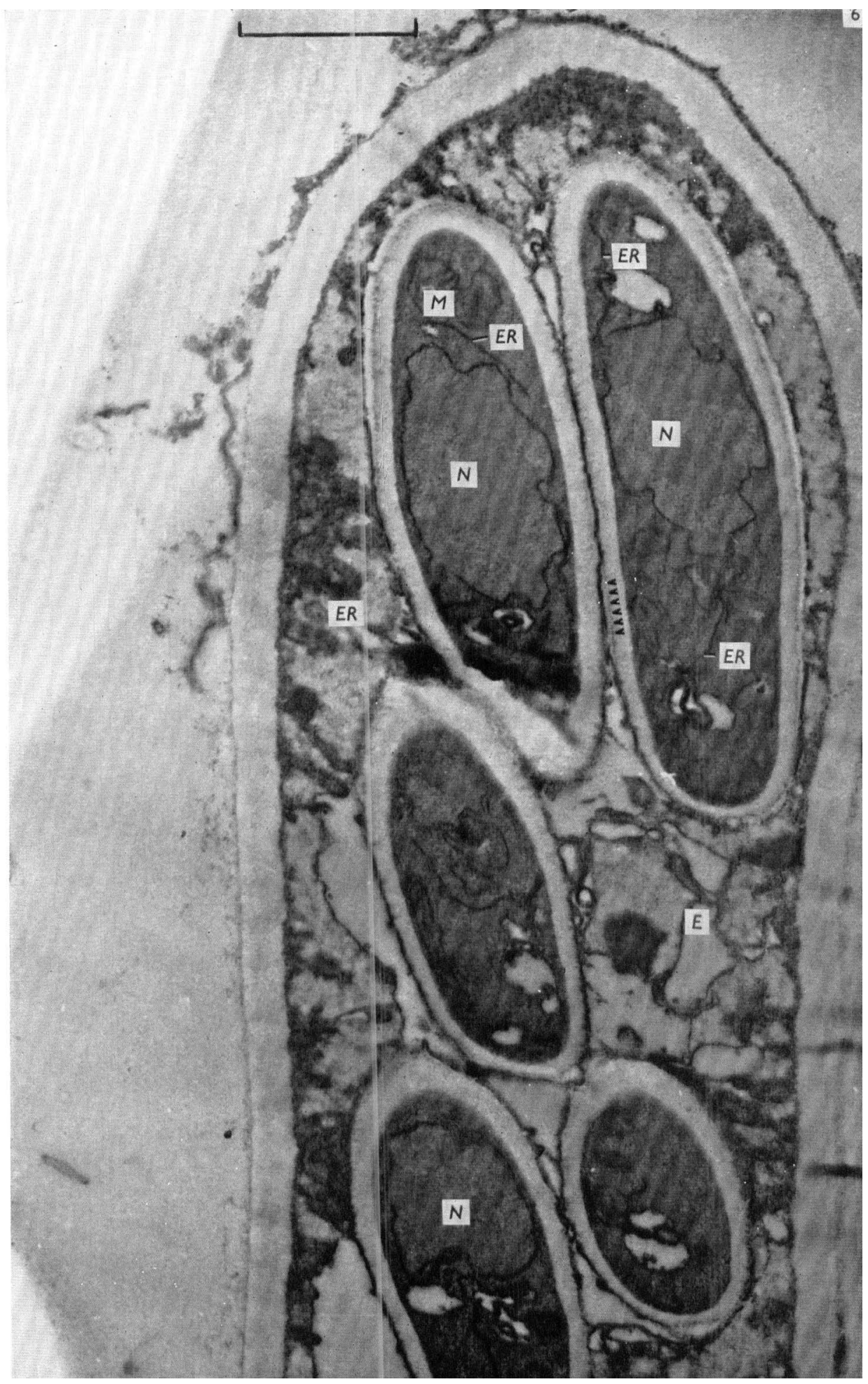

R. T. MOORE AND J. H. MCALEAR 
of a secondary spore coat, production of septa, nuclear multiplication, budding and fragmentation, and spore elongation; but we feel that the observations made and referred to here are typical and fundamental in ascospore formation.

This work was supported in part by grant H-3493 from the National Heart Institute, U.S. Public Health Service, and in part by a research fellowship, 9197-Cl, to R.T.M. from the National Institute of Allergy and Infectious Diseases, U.S. Public Health Service, and sponsored by Professor R. P. Korf.

This paper is No. 6 in a series on the Fine Structure of Mycota.

\section{REFERENCES}

Edwards, G. A. \& Edwards, M. R. (1960). The intracellular membranes of Blastomyces dermatitidis. Amer. J. Bot. 47, 622.

MCAlear, J. H. \& Edwards, G. A. (1959). Continuity of plasma membrane and nuclear membrane. Exp. Cell Res. 16, 689.

Moore, R. T. (1962). Fine structure of mycota. 1. Electron microscopy of the discomycete Ascodesmis. Nova Hedwigia (in the Press).

Moore, R. T. \& McAlear, J. H. (1961). Fine structure of mycota. 3. Reconstruction from skipped serial sections of the nuclear envelope and its continuity with the plasma membrane. Exp. Cell Res. 24, 588.

\section{EXPLANATION OF PLATES}

Asci of Dasyscyphus sp. showing progressive steps in ascospore initiation and maturation. $E$, epiplasm; $L R$, endoplasmic reticulum; $M$, mitochondria; $N$, nucleus. Scale lines equal $1 \mu$.

\section{Plate 1}

Fig. 1. Fusion nucleus. The nuclear membrane is still present and the nucleoplasm shows typical light, coarse granular, and dark, fine granular regions. Approx. $\times 50,000$.

Fig. 2. An early stage in meiosis. The nuclear envelope has dissociated and the nucleophase merges into the cytoplasm. Approx. $\times 30,000$.

\section{Plate 2}

Fig. 3. A stage considered to be later than fig. 2, The two masses composed of light and dark moieties are interpreted to be nuclei; a membrane fragment near the lower aggregate (wedges) may be a segment of the dissociated nuclear envelope. Approx. $\times 25,000$.

Fig. 4. A later stage showing re-formation of nuclear and endoplasmic reticulum endomembranes. Approx. $\times 30,000$.

\section{Plate: 3}

Fig. 5. A still later stage believed to represent late telophase. Re-formation of the nuclear membranes is nearly complete, but that nuclear separation has not yet begun is suggested by the opposing gaps in the respective nuclear envelopes (arrows). The endoplasmic reticulum is prominent and has become more highly organized. Approx. $\times 50,000$.

\section{Plate 4}

Fig. 6. A nearly mature ascus. Walls have encapsulated the nuclei and portions of the ascus cytoplasm; the epiplasm, cytoplasm left outside the ascospores, is partially broken down. The spores contain what may be interpreted as typical nuclei, mitochondria and endoplasmic reticulum; the last mentioned appears to form continuities between the nucleus and the plasma membrane in the upper right spore. Each spore is bounded by a dark layer of material that appears to be separable from the spore wall (wedges). Approx. $\times \mathbf{2 5 , 0 0 0 .}$ 